\title{
Calvarial Fracture Patterns on CT Imaging Predict Risk of a Delayed Epidural Hematoma following Decompressive Craniectomy for Traumatic Brain Injury
}

\author{
J.F. Talbott, A. Gean, E.L. Yuh, and S.I. Stiver
}

\begin{abstract}
BACKGROUND AND PURPOSE: The development of a delayed epidural hematoma as a result of decompressive craniectomy represents an urgent and potentially lethal complication in traumatic brain injury. The goal of this study was to determine the incidence of delayed epidural hematoma and whether patterns of skull fractures on the preoperative CT scan could predict risk of a delayed epidural hematoma.
\end{abstract}

\begin{abstract}
MATERIALS AND METHODS: We retrospectively evaluated medical records and imaging studies for patients with acute traumatic brain injury who underwent a decompressive craniectomy during a 9-year period. We compared patterns of skull fractures contralateral to the side of the craniectomy with the occurrence of a postoperative delayed epidural hematoma.

RESULTS: In a series of 203 patients undergoing decompressive craniectomy for acute traumatic brain injury, the incidence of a delayed epidural hematoma complication was $6 \%$ (12 of 203). All 12 patients who developed a delayed epidural hematoma had a contralateral calvarial fracture on preoperative CT at the site where the delayed epidural hematoma subsequently formed. A contralateral calvarial fracture has perfect sensitivity (100\%) for subsequent development of delayed epidural hematoma in our study population. Moreover, a contralateral calvarial fracture involving 2 or more bone plates had an especially high diagnostic odds ratio of 41 for delayed epidural hematoma.
\end{abstract}

CONCLUSIONS: Recognition of skull fracture patterns associated with delayed epidural hematoma following decompressive craniectomy may reduce morbidity and mortality by prompting early postoperative intervention in high-risk situations.

ABBREVIATIONS: $\mathrm{DC}=$ decompressive craniectomy; $\mathrm{DEDH}=$ delayed epidural hematoma; $\mathrm{GOS}=$ Glasgow Outcome Scale; ICP = intracranial pressure; $\mathrm{TBI}=$ traumatic brain injury

D ecompressive craniectomy (DC) has been used with increasing frequency over the past few years to control raised intracranial pressure (ICP) that accompanies severe traumatic brain injury, malignant middle cerebral infarction, and other central nervous system conditions. ${ }^{1-4}$ While DC is a potentially life-saving operation, the procedure is associated with a number of complications. $^{5-7}$ One potential devastating complication following DC is the development of an epidural hematoma remote to the craniectomy. ${ }^{6,8-13}$ This hematoma has been termed a delayed epi-

Received January 22, 2014; accepted after revision March 29.

From the Department of Radiology and Biomedical Imaging (J.F.T., A.G., E.L.Y.) and Brain and Spine Injury Center (J.F.T., A.G., E.L.Y.), San Francisco General Hospital, San Francisco, California; and Department of Neurological Surgery (A.G., S.I.S.), University of California, San Francisco, California.

Please address correspondence to Jason Talbott, MD, PhD, San Francisco General Hospital, Department of Radiology, 1001 Potrero Ave, Room 1X55F, San Francisco, CA 94110; e-mail: Jason.talbott@ucsf.edu

三 Indicates article with supplemental on-line table.

http://dx.doi.org/10.3174/ajnr.A4001 dural hematoma (DEDH), as it is often first detected on the postoperative CT imaging studies. However, the hematoma may evolve during the decompression and present intraoperatively with external cerebral herniation through the craniectomy defect. ${ }^{8,11,12}$ Although initially considered rare, the DEDH has become more common in the last decade with the resurgence of DC as a means to control raised ICP following traumatic brain injury (TBI). Clinical findings associated with DEDH include intraoperative brain swelling, ${ }^{8}$ pupillary abnormalities, ${ }^{14}$ increased ICP measurements, and acute neurologic deterioration. ${ }^{5,15}$ The DEDH represents a potentially fatal complication and often requires a second surgical decompression. ${ }^{10,15}$

A frequent radiographic abnormality found in association with a DEDH is the presence of a calvarial skull fracture, contralateral to the craniectomy (Figs 1-3). ${ }^{8-13,16}$ In the case series of Su et al, ${ }^{8}$ contralateral calvarial fractures were present on the preoperative CT in 10 of 12 patients who developed a DEDH. ${ }^{8}$ In the remaining 2 patients, a radiographically occult fracture was identified intraoperatively during DEDH evacuation. Despite the po- 

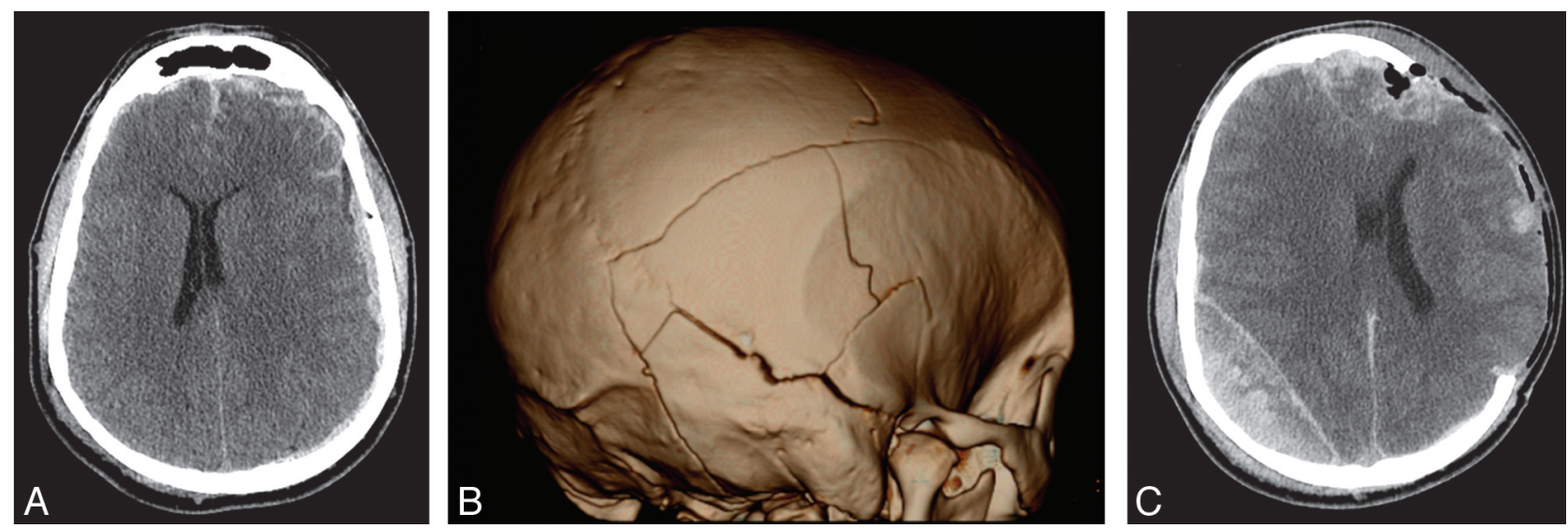

FIG 1. DEDH following decompressive hemicraniectomy for traumatic brain injury. A, Axial noncontrast CT at the level of the corona radiata before initial decompression demonstrates diffuse effacement of the cerebral sulci and small holohemispheric subdural hematoma on the left. Small bilateral frontal hemorrhagic contusions and scattered subarachnoid hemorrhage were also present. $B$, Three-dimensional volumerendered image reconstructed from bone algorithm noncontrast head CT demonstrates complex calvarial fractures on the right involving the right temporal, occipital, and parietal bones. C, Noncontrast head CT performed after left decompressive hemicraniectomy shows interval development of a new, large, heterogeneously attenuated epidural hematoma in the right parietal region with new right to left midline shift and left frontotemporal external herniation.

tential high clinical significance of this finding, whether varying fracture patterns are associated with different rates of DEDH in patients undergoing DC has not been studied. Such descriptions would be important as preoperative CT findings implying high risk for DEDH could alert the surgeon to the risks of intraoperative brain swelling through the craniectomy defect and also suggest a need for early postoperative CT imaging to monitor for this potentially fatal complication.

The present study retrospectively evaluated a cohort of 203 patients undergoing DC for acute TBI over a 9-year period. We characterized contralateral calvarial skull fractures in all patients undergoing DC. We compared fracture patterns in patients who did versus those who did not develop DEDH. The goal of this study was to determine the incidence of DEDH after DC and whether skull fracture patterns on the preoperative CT scan can predict risk of a DEDH.

\section{MATERIALS AND METHODS}

The study was approved by the Committee on Human Subjects Research and was Health Insurance Portability and Accountability Act compliant. Patients who presented to our Level 1 trauma center with acute TBI and who underwent DC were retrospectively identified from our radiologic database of imaging reports using the search terms "craniectomy" and "hemicraniectomy." The search spanned a 9-year period between 2001 and 2010. Patients undergoing DC for nontraumatic indications or for penetrating trauma, including gunshot wounds, were excluded. Bifrontal decompressive craniectomies were also excluded. There were no age limitations. The study cohort included all patients undergoing DC who met inclusion criteria. The DC surgical procedure entailed removal of a large portion of frontal-temporalparietal-occipital skull bone that measured approximately 12 by $15 \mathrm{~cm}$. The underlying dura was opened in a stellate fashion to the bone edges, and the scalp flap was closed over the decompressed brain without a duroplasty.

CT studies consisting of thin section $(0.625$ to $1.25 \mathrm{~mm})$ multidetector-row CT (Lightspeed VCT and CTi; GE Healthcare,
Milwaukee, Wisconsin) images with soft tissue and bone algorithm reconstructions were available for analysis on a PACS viewing station. CT imaging studies of the brain and calvaria were analyzed by 3 neuroradiologists with 4,8 , and over 20 years of experience. Review of the preoperative CT images was performed without knowledge of whether the patient developed a DEDH. The number and distribution of calvarial bone fractures on both sides of the skull were tabulated before review of the postoperative CT. Calvarial fractures were defined as those involving the parietal bone, squamosal temporal bone, calvarial sphenoid, calvarial occipital, and frontal bones. Skull base, nonsquamosal temporal bone, central sphenoid, and facial bone fractures were excluded from analysis. Preoperative CT images were scrutinized for epidural hematomas underlying calvarial skull fractures. In addition, the presence or absence of an acute subdural hematoma, signs of midline shift, and status of the basal cisterns were also recorded from the preoperative $\mathrm{CT}$ images. Cisterns were classified as open, effaced, or absent. ${ }^{17}$ Postoperative CT images were examined for evidence of a DEDH, contralateral or remote to the craniectomy skull defect, after review of the preoperative CT images was complete. Whether the DC had been performed on the coup versus contre coup side of injury was noted. The relationship between DEDHs and calvarial skull fractures was noted. The DEDH volume, maximum diameter, and midline shift on the postoperative CT were recorded. Volume calculations for DEDHs were based on the Petersen and Espersen equation (one-half $X$ anteroposterior diameter $\times$ transverse diameter $\times$ craniocaudal diameter in $\mathrm{cm}) .{ }^{18,19}$ Calvarial fracture patterns contralateral to the side of DC were compared between patients who developed versus those who did not develop a DEDH.

Clinical records for the subgroup of patients with complications of a DEDH were reviewed in detail. Patient and injury characteristics, including the preoperative Glasgow Coma Scale, indications for postoperative CT scanning, and time interval from close of surgery to the postoperative CT were collected. Indications for a postoperative CT scan were classified as routine, ele- 

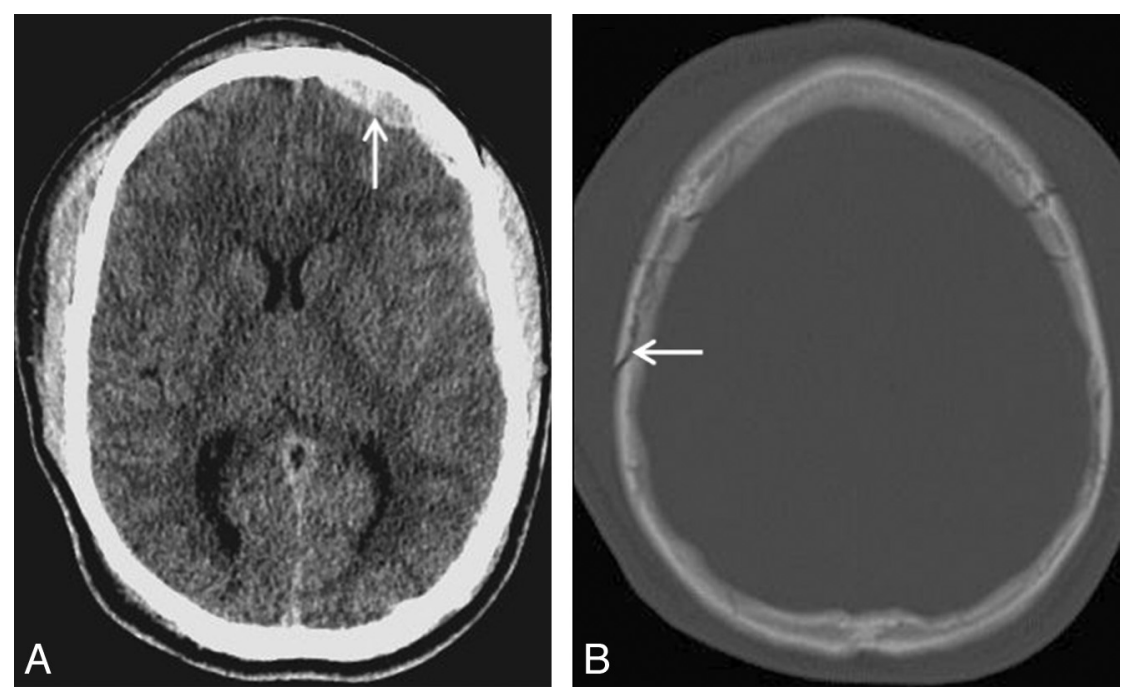

exact tests were used to determine whether there were significantly different rates of DEDH for different calvarial fracture patterns. A $P$-value $<.05$ was considered statistically significant. Statistical analyses were performed using SPSS version 18 statistical software (IBM, Armonk, New York).

\section{RESULTS}

Among 203 patients who underwent DC for acute nonpenetrating TBI, 12 patients (5.9\%) developed a de novo DEDH remote to the craniectomy site. In all cases, the preoperative CT scan was devoid of any signs of a pre-existing epidural hematoma in the location where the DEDH
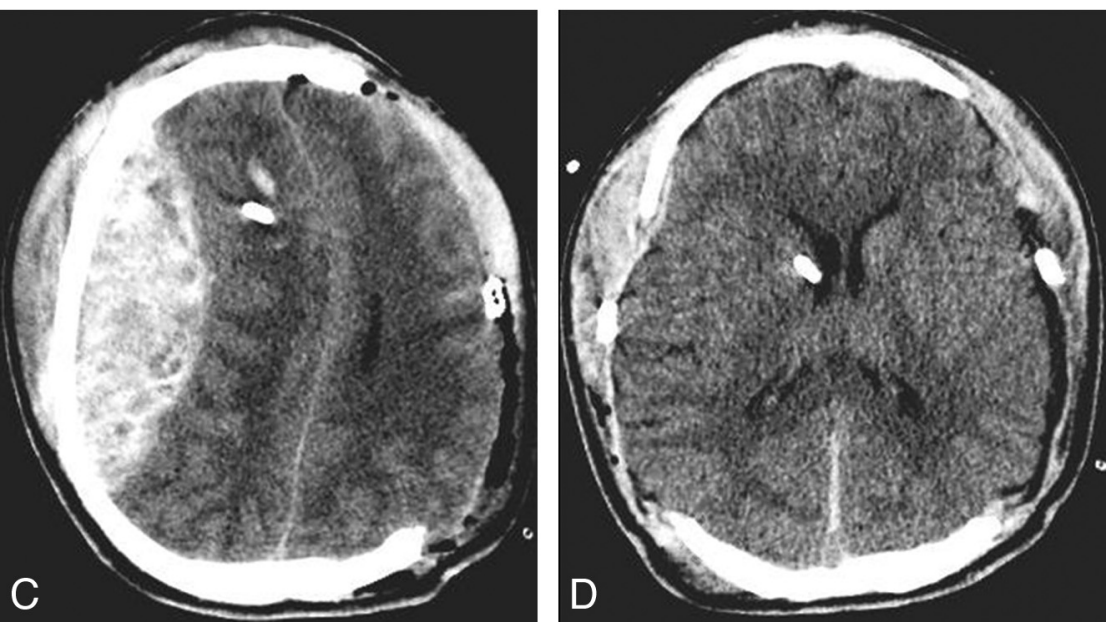

FIG 2. A, Axial noncontrast $C T$ at the level of the internal capsules demonstrates a left frontal extra-axial hematoma (arrow). There is also diffuse sulcal effacement. B, Nondisplaced rightsided parietal calvarial fracture is present on the preoperative CT (arrow). Right frontal and temporal calvarial fractures were also present (not shown). Coronal suture diastasis is seen on the left. C, Following left-sided decompressive craniectomy, a large, predominantly parietal, DEDH developed adjacent to the patient's right parietal bone fracture. $D, A$ decompressive craniectomy was subsequently performed on the right side with evacuation of DEDH and significant improvement in mass effect.

vated ICP, or clinical findings of a fixed, dilated pupil. We collected information pertaining to hospital course and patient outcome including whether the patient underwent re-operation for evacuation of the DEDH, hospital length of stay, disposition from hospital, and the Glasgow Outcome Scale (GOS) at time of discharge. The GOS is assigned on a scale of 1 to 5 , with ratings of 1 (death), 2 (persistent vegetative), 3 (severe disability), 4 (moderate disability), and 5 (good outcome). ${ }^{20,21}$ Outcome was dichotomized into favorable (GOS 4 and 5) and unfavorable (GOS 1-3) outcomes.

Continuous data, conforming to a normal distribution curve, were described using mean values \pm standard deviation. The Kolmogorov-Smirnov test was used to test whether a variable fitted a normal distribution curve. Categoric variables are presented as percentages. Sensitivity, specificity, and diagnostic odds ratios for calvarial fracture patterns were calculated from counts of the numbers of patients with positive and negative test results for an identifying criterion using standard statistical formulae. Fisher later developed. Patient characteristics, injury mechanism, hospital course, and outcome results are summarized in Online Table 1. This patient subgroup is predominantly composed of men (10 of 12 , $83 \%)$ with a mean age of $32.3 \pm 13.5$ years (range 15-57 years). Two-thirds (8 of 12, $67 \%$ ) of the patients with a DEDH met criteria for a severe TBI (preoperative Glasgow Coma Scale $\leq 8$ ) with a mean preoperative Glasgow Coma Scale score of $7.0 \pm 3.3$. Mechanisms of injury involved high impact injuries without head protection in 10 of 12 (83\%) patients. Assaults, mediated by generally lower energy contact forces, were observed in only $2(17 \%)$ patients.

Preoperative CT imaging findings are summarized in On-line Table 2. The coup site was identified in 9 patients (75\%), and the decompressive craniectomy was noted to have been performed on the contre coup side in these 9 patients. Subgaleal scalp swelling was bilateral in $3(25 \%)$ of

12 patients, obscuring assignment of the craniectomy to the coup versus contre coup side. Acute subdural hematomas ipsilateral to the side of the decompressive craniectomy were present in all 12 patients who developed a DEDH. These acute subdural hematomas measured an average thickness of $5.8 \pm 2.6 \mathrm{~mm}$ with midline shift of $6.4 \pm 3.8 \mathrm{~mm}$. In 9 of $12(75 \%)$ patients, the subdural hematoma was categorized as "complex," as evidenced by signs of mass effect in proportion to the thickness of the subdural hematoma. Basal cisterns were compressed in 4 of 12 (33\%) patients and completely obliterated in 7 of $12(58 \%)$ patients undergoing DC. Together, signs of raised ICP as judged by midline shift greater than $5 \mathrm{~mm}$ and/or obliterated status of the basal cisterns were present in 11 of $12(92 \%)$ patients.

The time interval from completion of the decompressive surgery to the postoperative CT imaging averaged $12.8 \pm 17.1$ hours. Indications for obtaining a follow-up CT after DC included elevated ICP $(n=5)$, new pupillary deficit $(n=3)$, and routine postoperative surveillance $(n=4)$. Imaging characteristics of the 

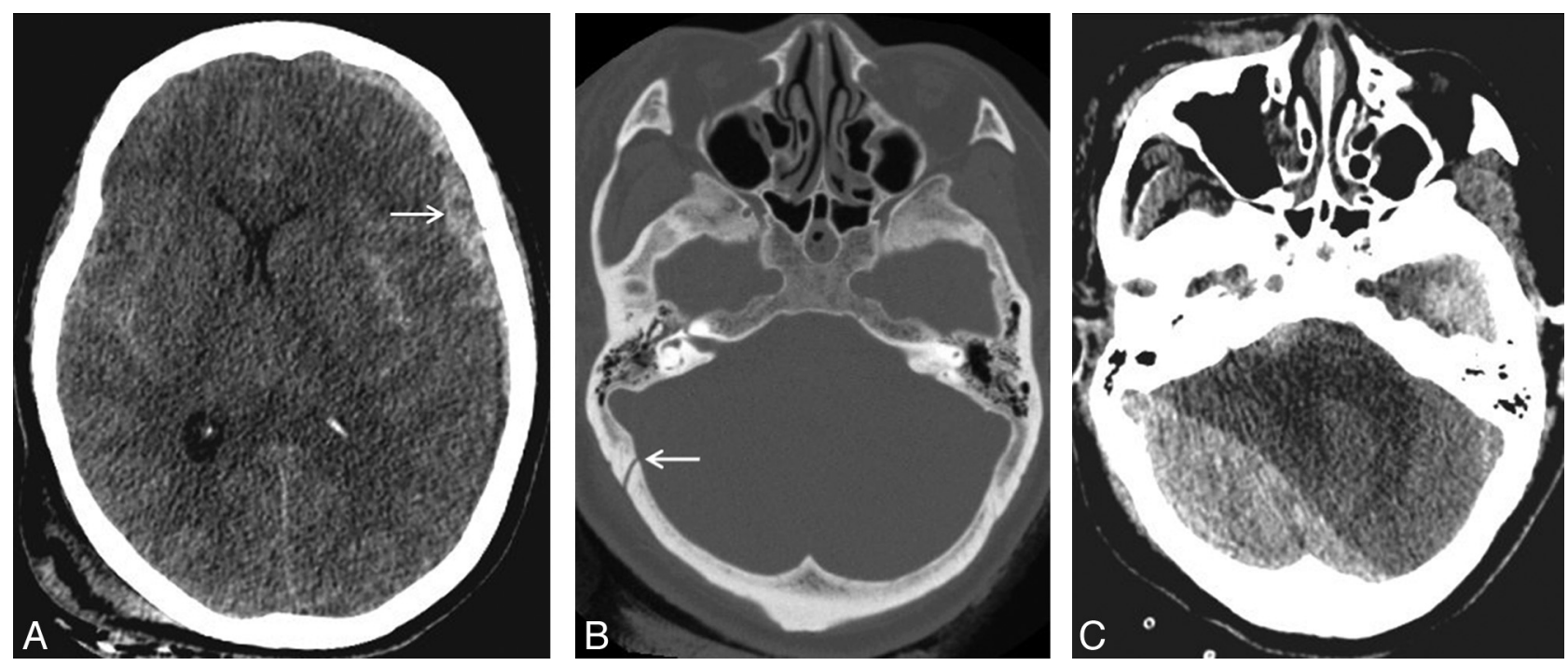

FIG 3. A, Axial noncontrast CT at the level of the internal capsules demonstrates a left-sided frontoparietal subdural hematoma (arrow). There is also bilateral subarachnoid hemorrhage, diffuse sulcal effacement, left to right midline shift, and near complete effacement of the left lateral ventricle with early trapping of the right lateral ventricle. $B$, Nondisplaced right occipital calvarial fracture paralleling the ipsilateral lambdoid suture is also identified on the preoperative head CT. A right sided parietal calvarial fracture was also present (not shown). C, Following left-sided decompressive craniectomy, postoperative head CT reveals a large right-sided occipital DEDH in the posterior fossa, adjacent to right-sided occipital bone fracture, with severe mass effect on the cerebellum and brain stem and effacement of the fourth ventricle.

Sensitivity, specificity, and diagnostic odds ratio analyses of contralateral calvarial skull fractures for DEDHs after DC

\begin{tabular}{lccc}
\hline & $\begin{array}{c}\text { Any } \\
\text { Contralateral } \\
\text { Calvarial Fracture } \\
(\boldsymbol{n}=\mathbf{5 5})\end{array}$ & $\begin{array}{c}\text { Fractures } \\
\text { Involving a } \\
\text { Single Bone } \\
\text { Plate }(\boldsymbol{n}=\mathbf{3 3})\end{array}$ & $\begin{array}{c}\text { Fractures } \\
\text { Involving } \geq 2 \\
\text { Bone Plates } \\
(\boldsymbol{n}=\mathbf{2 2})\end{array}$ \\
\hline Sensitivity & $100 \%$ & $25 \%$ & $75 \%$ \\
$\begin{array}{l}\text { Specificity } \\
\begin{array}{l}\text { Diagnostic odds } \\
\text { ratio }\end{array}\end{array}$ & $\begin{array}{c}\text { Undefined } \\
\text { a }\end{array}$ & $83 \%$ & $94 \%$ \\
\hline
\end{tabular}

a The sensitivity of the finding "any calvarial fracture" is 100\%, therefore false negatives $=0$ and odds ratio is undefined.

DEDHs are summarized in On-line Table 2. Postoperative DEDHs were remarkable for their large size (mean volume $=$ $86.0 \pm 67.2 \mathrm{~mL}$; mean thickness $24.8 \pm 9.8 \mathrm{~mm}$ ) and the degree of associated midline shift (mean $9.5 \pm 7.0 \mathrm{~mm}$ ). The location of DEDHs was contralateral to the side of craniectomy in 10 of 12 $(83 \%)$ of patients. In 2 patients, DEDHs were bilateral, underlying vertex fractures that crossed the midline.

The locations and patterns of skull fractures for all patients undergoing DC are summarized in On-line Table 3. Preoperative CT imaging identified a calvarial fracture contralateral to the planned craniectomy in 55 of 203 patients with DC (27\%). In all 12 patients who developed a $\mathrm{DEDH}$, a calvarial fracture(s) was present at the site where the DEDH formed. Fractures contralateral to the DC were confined to a single calvarial bone in $60 \%$ (33 of 55) and involved 2 or more calvarial bones in 40\% (22 of 55) of patients. Sensitivity, specificity, and diagnostic odds ratio values for the different fracture patterns in relation to occurrence of DEDHs are summarized in the Table. Even though all 12 patients with DEDH had a skull fracture (sensitivity 100\%) contralateral to the side of DC, only 3 of these fractures (25\%) were limited to a single bone plate. In contrast, the sensitivity of fractures that spanned 2 or more bone plates was $75 \%$. Statistical analysis demonstrated a highly significant association $(P=.005$; Fisher exact test) between development of a DEDH and contralateral fractures involving 2 or more calvarial bones.

Reoperation was performed in 8 of the $12(67 \%)$ patients who developed a DEDH. In 7 of these 8 patients, DEDHs exceeded the guidelines for surgical evacuation of epidural hematomas in closed TBI (volume over $30 \mathrm{~mL}$ with greater than $5 \mathrm{~mm}$ of midline shift). ${ }^{22}$ Mean Glasgow Coma Scale in the reoperated group of patients was $7.3 \pm 3.6$ and mean time to acquisition of the postoperative CT was $7.0 \pm 7.0$ hours. In the reoperated group of 8 patients, the mean DEDH volume was $105.3 \pm 65.3 \mathrm{~mL}$, diameter thickness $27.9 \pm 9.3 \mathrm{~mm}$, and midline shift $11.4 \pm 7.2 \mathrm{~mm}$. One patient had a small DEDH without midline shift; however, intraparenchymal swelling with development of a dilated, fixed pupil on the side of the DEDH prompted a decompressive craniectomy together with evacuation of the small DEDH. This patient ultimately had a persistent vegetative outcome. Five patients underwent reoperation for DEDHs larger than $100 \mathrm{~mL}$ (mean volume $148.2 \pm 31.6 \mathrm{~mL}$, thickness $33 \pm 5.9 \mathrm{~mm}$, and midline shift $15 \pm$ $4.9 \mathrm{~mm}$ ). In the group of 12 patients with DEDH, 4 patients did not undergo reoperation. In 3 of these 4 patients, the volume of the DEDH was less than $30 \mathrm{~mL}$. One patient, not reoperated upon, had a large DEDH (137 mL) with $13 \mathrm{~mm}$ midline shift, but the patient's neurologic condition was deemed nonsurvivable.

Outcome was analyzed for all 12 patients with a DEDH, managed operatively and nonoperatively. The mean hospital length of stay in this group was $45.6 \pm 52.9$ days. Two patients died within 48 hours of admission. At the time of hospital discharge, outcome was favorable (GOS 4 or 5) in 4 of 12 (33\%), and unfavorable (GOS $1-3)$ in 8 of $12(67 \%)$ patients. In the 8 patients undergoing reoperation for their DEDH, 5 survived (63\%) and favorable outcome was achieved in 2 ( 2 of $8,25 \%)$. Reoperation for the 5 patients with large DEDH over $100 \mathrm{~mL}$ yielded 2 survivors ( 2 of 5 , $40 \%$ ), with Glasgow Coma Scale outcomes of 4 and 3 at time of hospital discharge.

AJNR Am J Neuroradiol 35:1930-35 Oct 2014 www.ajnr.org 1933 


\section{DISCUSSION}

As randomized studies strive to define the role of DC in the management of acute TBI, complications directly related to this procedure have become increasingly important. ${ }^{2,5-7,13,23-27}$ Complications of a DEDH arise from relief of the tamponade effect and hemorrhagic expansion of an injured meningeal artery, dural vein, or fractured diploe, upon opening of the skull. We observed a $6 \%$ incidence of DEDH following DC for acute TBI. This is comparable with the $4.5 \%$ incidence of DEDH reported by Matsuno et al. ${ }^{10}$ Wen et $\mathrm{al}^{13}$ recently published the largest cohort to date of 15 patients with TBI developing delayed extra-axial hemataoma contralateral to decompressive craniectomy, with a reported incidence of $6.8 \%$, very similar to our incidence results. In our analysis of 203 preoperative CT scans, the finding of any contralateral calvarial skull fracture had a positive predictive value of $22 \%$ and perfect sensitivity (100\%) for later development of a DEDH. Furthermore, the number of bone plates involved by the skull fracture was important. Specifically, if 2 or more calvarial bones are fractured on the side contralateral to craniectomy, the diagnostic odds ratio for developing DEDH is 41 .

Our study of 12 patients with DEDH complications revealed a number of clinically significant observations. First, after removal of a large portion of the skull, the brain can accommodate large DEDHs with significant degrees of midline shift, without progression to brain death. In our study, only 2 of 12 patients with DEDH met clinical criteria for brain death. Second, patients can harbor DEDH of considerable size without clinical signs. In 2 patients, a DEDH measuring over $100 \mathrm{~mL}$ was first detected postoperatively by routine surveillance CT imaging. Third and most importantly, survival and good outcomes may be achieved by reoperation for evacuation of DEDHs. In our study, survival and outcomes following reoperation of even large DEDHs over $100 \mathrm{~mL}$ exceeded expectation given the large size and midline shift of these lesions. Confirmation of this trend with larger patient cohorts is warranted. Takeuchi et $\mathrm{al}^{28}$ observed that CT scans can identify most new postoperative lesions even when performed within 1 hour after surgery for TBI. Thus, recognition of calvarial fracture patterns highly associated with DEDH may allow selection of patients for early postoperative CT scanning to reduce morbidity and mortality associated with DEDH complications after DC.

Our study has several limitations, including its retrospective study design. Over the 9-year span of the study, radiologic imaging protocols and patterns and standards of patient care may well have varied and are sources of potential bias and error. In addition, the small sample of only $12 \mathrm{DEDH}$ lesions limited more detailed statistical analysis of this patient subgroup. Our study is also limited by the lack of long-term patient follow-up and outcome data.

\section{CONCLUSIONS}

The presence of a calvarial fracture contralateral to the anticipated side of craniectomy should alert the radiologist and neurosurgeon to the risk of a DEDH complication. This prepares the neurosurgeon for the possibility of intraoperative brain swelling through the craniectomy defect, and identifies high-risk patients in whom an early postoperative surveillance CT scan may be warranted to circumvent devastating neurologic injury from DEDH. Calvarial fractures involving 2 or more bone plates are particularly highly predictive of DEDH. Recognition of skull fracture patterns associated with DEDH may help to identify patients at risk and prompt closer imaging monitoring to detect DEDH earlier.

Disclosures: Alisa Gean-UNRELATED: Board Membership: www.image32.com, Comments: no financial compensation yet; Consultancy: National Football League (NFL); Employment: www.hindsightwines.com (owner); Expert Testimony: various legal firms (plaintiff and defense); Payment for Lectures and the Development of Educational Presentations: Educational Symposia (various courses and meetings, Tampa, Florida); Ceretom (Samsung); Royalties: Lippincott Williams \& Wilkins, Comments: author, Brain Injury: Applications from War and Terrorism; Stock/Stock Options: GBLI (Global Indemnity), NASDAQ, and Ceretom (Samsung); Travel/Accommodations/Meeting Expenses Unrelated to Activities Listed: honoraria, University of California, San Francisco.

\section{REFERENCES}

1. Hutchinson P. Decompressive craniectomy for traumatic brain injury. Can J Neurol Sci 2011;38:541-42

2. Hutchinson PJ, Kirkpatrick PJ. Decompressive craniectomy in head injury. Curr Opin Crit Care 2004;10:101-04

3. Di Rienzo A, Iacoangeli M, Rychlicki F, et al. Decompressive craniectomy for medically refractory intracranial hypertension due to meningoencephalitis: report of three patients. Acta Neurochir (Wien) 2008;150:1057-65

4. Hofmeijer J, Kappelle LJ, Algra A, et al. Surgical decompression for space-occupying cerebral infarction (the Hemicraniectomy After Middle Cerebral Artery infarction with Life-threatening Edema Trial [HAMLET]): a multicentre, open, randomised trial. Lancet Neurol 2009;8:326-33

5. Stiver SI. Complications of decompressive craniectomy for traumatic brain injury. Neurosurg Focus 2009;26:E7

6. Yang XF, Wen L, Shen F, et al. Surgical complications secondary to decompressive craniectomy in patients with a head injury: a series of 108 consecutive cases. Acta Neurochir (Wien) 2008;150:1241-47

7. Honeybul S. Complications of decompressive craniectomy for head injury. J Clin Neurosci 2010;17:430-35

8. Su TM, Lee TH, Chen WF, et al. Contralateral acute epidural hematoma after decompressive surgery of acute subdural hematoma: clinical features and outcome. J Trauma 2008;65:1298-302

9. Mohindra S, Mukherjee KK, Gupta R, et al. Decompressive surgery for acute subdural haematoma leading to contralateral extradural haematoma: a report of two cases and review of literature. $\mathrm{Br} \mathrm{J} \mathrm{Neu}$ rosurg 2005;19:490-94

10. Matsuno A, Katayama H, Wada H, et al. Significance of consecutive bilateral surgeries for patients with acute subdural hematoma who develop contralateral acute epi- or subdural hematoma. Surg Neurol 2003;60:23-30

11. Meguro K, Kobayashi E, Maki Y. Acute brain swelling during evacuation of subdural hematoma caused by delayed contralateral extradural hematoma: report of two cases. Neurosurgery 1987;20: 326-28

12. Feuerman T, Wackym PA, Gade GF, et al. Intraoperative development of contralateral epidural hematoma during evacuation of traumatic extraaxial hematoma. Neurosurgery 1988;23:480-84

13. Wen L, Li QC, Wang SC, et al. Contralateral haematoma secondary to decompressive craniectomy performed for severe head trauma: a descriptive study of 15 cases. Brain Inj 2013;27:286-92

14. Moon KS, Lee JK, Kim TS, et al. Contralateral acute subdural hematoma occurring after removal of calcified chronic subdural hematoma. J Clin Neurosci 2007;14:283-86

15. Su TM, Lee TH, Huang YH, et al. Contralateral subdural effusion after decompressive craniectomy in patients with severe traumatic brain injury: clinical features and outcome. J Trauma 2011;71: 833-37

16. Lasierra JL, Fuentes CG, Vazquez DT, et al. Contralateral extraaxial hematomas after urgent neurosurgery of a mass lesion in patients 
with traumatic brain injury. Eur J Trauma Emerg Surg 2013; 39:277-83

17. Maas AI, Hukkelhoven CW, Marshall LF, et al. Prediction of outcome in traumatic brain injury with computed tomographic characteristics: a comparison between the computed tomographic classification and combinations of computed tomographic predictors. Neurosurgery 2005;57:1173-82

18. Petersen OF, Espersen JO. Extradural hematomas: measurement of size by volume summation on CT scanning. Neuroradiology 1984; 26:363-67

19. Bullock MR, Chesnut R, Ghajar J, et al. Appendix I: post-traumatic mass volume measurement in traumatic brain injury patients. $\mathrm{Neu}$ rosurgery 2006;58:S2-61

20. Wilson JT, Pettigrew LE, Teasdale GM. Structured interviews for the Glasgow Outcome Scale and the extended Glasgow Outcome Scale: guidelines for their use. J Neurotrauma 1998;15:573-85

21. Jennett B, Bond M. Assessment of outcome after severe brain damage. Lancet 1975;1:480-84
22. Bullock MR, Chesnut R, Ghajar J, et al. Surgical management of acute epidural hematomas. Neurosurgery 2006;58:S7-15

23. Hutchinson PJ, Kirkpatrick PJ. Craniectomy in diffuse traumatic brain injury. N Engl J Med 2011;365:375

24. Timmons SD, Ullman JS, Eisenberg HM. Craniectomy in diffuse traumatic brain injury. N Engl J Med 2011;365:373

25. Cooper DJ, Rosenfeld JV, Murray L, et al. Decompressive craniectomy in diffuse traumatic brain injury. N Engl J Med 2011; 364:1493-502

26. Plesnila N. Decompression craniectomy after traumatic brain injury: recent experimental results. Prog Brain Res 2007;161: 393-400

27. Sahuquillo J, Arikan F. Decompressive craniectomy for the treatment of refractory high intracranial pressure in traumatic brain injury. Cochrane Database Syst Rev 2006:CD003983

28. Takeuchi S, Takasato Y, Suzuki G, et al. Postoperative computed tomography after surgery for head trauma. J Trauma Acute Care Surg 2012;73:1254-60 